\title{
Original
}

\section{Systematic determination of thyroid volume by ultrasound examination from infancy to adolescence in Japan: The Fukushima Health Management Survey}

\author{
Satoru Suzuki 1), 2), Sanae Midorikawa ${ }^{1), 3)}$, Toshihiko Fukushima ${ }^{1), 2)}$, Hiroki Shimura ${ }^{1), 4)}$, \\ Tetsuya Ohira ${ }^{1), 5)}$, Akira Ohtsuru ${ }^{1), 3)}$, Masafumi Abe ${ }^{1)}$, Yoshisada Shibata ${ }^{1)}$, Shunichi Yamashita ${ }^{1)}$ and \\ Shinichi Suzuki ${ }^{1), 2)}$ of the Thyroid Examination Unit of the Radiation Medical Science Center for the \\ Fukushima Health Management Survey \\ 1) Radiation Medical Science Center for the Fukushima Health Management Survey, Fukushima Medical University, Japan \\ 2) Department of Thyroid and Endocrinology, School of Medicine, Fukushima Medical University, Japan \\ 3) Department of Radiation Health Management, School of Medicine, Fukushima Medical University, Japan \\ 4) Department of Laboratory Medicine, School of Medicine, Fukushima Medical University, Japan \\ 5) Department of Epidemiology, School of Medicine, Fukushima Medical University, Japan
}

\begin{abstract}
Although several reports have defined normal thyroid volume depending on either age or body surface, there are no sequential reference values on childhood thyroid volume evaluated by using ultrasonography and epidemiological analysis in Japan. The aim of the present study was to establish updated reference values for thyroid volume by ultrasound examination and epidemiological analysis in 0-19 year-old Japanese children. It is based on a cross-sectional study conducted from October 9, 2011 to March 31, 2012. The subjects were 38,063 children who were examined by ultrasonography as the initial preliminary survey of the Fukushima Health Management Survey in October 9, 2011 to March 31, 2012. The width, thickness, and height of each lobe were measured and the volume of each lobe was calculated by the mean of the elliptical shape volume formula. The values of thyroid volume at the 2.5 and 97.5 percentiles of age and body surface area for each gender group were obtained from 0-19 year-old children. Positive correlation was observed between thyroid volume and either age or body surface. The right lobe was significantly larger than the left lobe. The thyroid volume in females was larger than that in males after adjusting body surface area. The reference values of childhood thyroid for each age or body surface area were obtained by this extensive survey using ultrasound. These reference values may be used to define the normal size of thyroid gland by echosonography in Japanese children, although thyroid volume may be affected by dimorphic factors such as sex hormones.
\end{abstract}

Keywords: Thyroid volume, Goiter, Body surface area, Ultrasonography, Health survey

THYROID hormone is crucial to brain development and bone growth, especially in the neonatal period as well as in further developmental periods such as infant, toddler, schoolchild, and adolescent. Early detection of thyroid illness is thus clinically important to prevent irreversible retardation during these periods. Although neonatal thyroid screening for congenital hypothyroidism is performed in many countries, it is hoped that

Submitted Oct. 16, 2014; Accepted Dec. 8, 2014 as EJ14-0478

Released online in J-STAGE as advance publication Jan. 15, 2015

Correspondence to: Satoru Suzuki, M.D., Ph.D., Department of Thyroid and Endocrinology, Fukushima Medical University School of Medicine, 1 Hikarigaoka, Fukushima City, Fukushima 9601295, Japan. E-mail: suzukisa@fmu.ac.jp thorough periodic thyroid examinations during childhood could be used to uncover conditions such as goiter, mild or asymptomatic hypothyroidism due to ectopic or hypoplastic thyroid gland, disturbance of thyroid hormone production, and pituitary dysfunction.

There is no doubt that routine serum sampling reveals precise thyroid function including FT3, FT4, $\mathrm{TSH}$, titer of thyroid related antibodies, and thyroglobulin concentration. The sampling itself, however, is not always acceptable to children, because they usually are averse to being pricked in the skin during blood withdrawal. By contrast, ultrasound is more acceptable for small children because the procedure is gentle and not invasive or painful. Ideally, the detection 
of enlarged or hypoplastic thyroid by ultrasonography would be useful for screening subclinical or clinical hyper- or hypothyroid in asymptomatic thyroid diseases in children.

After the development of ultrasonography, it was possible to calculate thyroid volume precisely and assess the size of the gland extensively. Once precise normal thyroid volume was accurately establsihed, it was described and reviewed in comparison with several reports on it by Kharchenko et al. [1]. Thyroid volume was assessed in relation to body surface area, age, thoracic circumference. etc., and several reference values were demonstrated with thyroid volume being calculated in several ways including direct measurement by mechanical equipment. Normal thyroid size as well as problems regarding quality of ultrasound morphometry including thyroid shape, calculation of thyroid size, and topography, were precisely described by Parshin et al. [2], along with normal reference values for age.

In a study of Chernobyl, goiter was defined with a calculation based on the mass data of thyroid volume measurement [3]. This study itself may have observed only a part of the full spectrum of meaning for the pathological goiter, since goiters abnormalities may be caused by a number of genetic, immunological, and nutritional factors. Meanwhile, the physiological meaning of "goiter" is simply defined as a large thyroid gland. The data obtained from the Chernobyl study were further used in an attempt to clarify the relationship between radiation exposure and thyroid volume changes in Belarus [4]. Ueda demonstrated normal thyroid volume calculated from the measurement of 300 thyroid glands in children [5]. However, there are no updated reference values of thyroid volume based on the three determinations of width, thickness, and height by ultrasonography in a wide age range and body surface area in children living in Japan, an iodine-rich country.

From these aspects, we have provided reference values for the measurement of thyroid volume by using a mass ultrasonographic survey and estimated the factors that affect thyroid volume from epidemiological insights through the Fukushima Health Management Survey.

\section{Methods and Subjects}

\section{Primary thyroid ultrasound examination}

Subjects of the thyroid ultrasound examination of the Fukushima Health Management Survey included approximately 360,000 children who were living in Fukushima Prefecture at the time of the nuclear accident and were aged 18 years or younger on March 11, 2011 [6]. The study subjects were 38,063 children who were examined in an initial preliminary survey during the period from Oct 9, 2011 to March 31, 2012. Survey population included residents of the towns of Kawamata, Namie, Futaba, Tomioka, Naraha, Hirono, and Okuma; the villages of Iitate, Kawauchi, Katsurao; and the cities of Minamisoma, Tamura, and Date, with a total census of 47,730 in March 2011. Participation rates were $79.7 \%$ for ages $0-19$ at the time of the study. This survey was approved by the ethical review committee of Fukushima Medical University (No. 1318). Written informed consent was obtained from the parents of the children surveyed. Ultrasound volumetric examination was performed using a commercial ultrasound machine, equipped with a linear array $10-12 \mathrm{MHz}$ probe. Length of the probe used was $40 \mathrm{~mm}$. Width, thickness and height of each lobe were measured and the volume of each lobe was calculated by the mean of the elliptical shape volume formula $(\pi / 6 \times$ width $\times$ thickness $\times$ height). In addition, any ultrasound abnormality observed during the examination was reported. Height and weight of each child were also measured to evaluate the volume of the gland in relation to body surface of the child. Among all subjects, some determinations of the measurement were not provided in 3,823 cases because of invalid information or difficulty in measurement. Eleven cases had developed hemiagenesis of thyroid gland. In total, 34,227 (male, 17,233; female, 16,994) subjects were completely examined and used in this study. The age (mean \pm SD) of the eliminated samples $(5.2 \pm 5.0)$ was significantly lower than that in the analyzed group (10.9 \pm 4.9$)$, although there was no gender difference.

\section{Statistical Analyses}

SPSS version 19 (SPSS Inc., Chicago, IL) was used for statistical analyses. Volume difference between right and left lobes was examined by paired t-test. Gender difference was analyzed by analysis of covariance (ANCOVA). The $p$-value refers to ANCOVA, adjusted for body surface area. As the body surface area was closely related to the age when it was surveyed (Pearson's correlation coefficient: 0.776), we have eliminated age as a covariate when ANCOVA was performed. 


\section{Results}

As shown in Table $1 \mathrm{a}$ and $\mathrm{b}$, over 300 cases were examined at any age period in males and females except for age of 0 , because of limitation in examination. Reference values (2.5 and 97.5 percentile) of thyroid size and volume measured by ultrasound, expressed as a normal range according to sex and age, and to sex and body surface area in sample of 0-19 year-old children, are shown in Table $1 \mathrm{a}$ and $\mathrm{b}$, respectively. The reference ranges are depicted in Fig. 1. The values at the 90 percentile increased exponentially during the period from 0 to 10 years in both the right or left thyroid lobe in both genders (Fig. $1 \mathrm{a}, \mathrm{b}$ ). Over the next several years, the growth rate gradually reduced and the range between the 10 and 90 percentiles extended annually in an age-dependent manner. The values at the 90 percentile reached a plateau at an age range of 16-19 years in both genders, while the values at the 90 percentile increased linearly with increasing body surface area in both the right or left lobes of the thyroid gland in both genders (Fig. $1 \mathrm{c}, \mathrm{d}$ ).

As shown in Table 2, the thyroid volume of the right lobe was significantly larger than that of the left lobe. ANCOVA indicated that thyroid volume in females was significantly larger than that in males after adjusting for body surface area (Table 3).

\section{Discussion}

Thyroid gland volume is reported to increase ageor body surface dependently [7]. The World Health Organization and International Council for Control of Iodine Deficiency Disorders demonstrated the normative values for thyroid volume in children aged 6-15 years [8]. In an iodine replete area, thyroid volume was inversely related to the median urinary iodide [9]. To screen for iodide deficiency disorders, international reference values were established [10]. Among 6 regions in the world, children in Japan, as representatives of the Asian region, had both the largest age-adjusted thyroid volumes and the largest body surface area-adjusted thyroid volumes. After publishing these data, recent studies on thyroid volume confirmed the international reference values although the number of cases are small (approximately 200-5000 cases) in all studies [1116]. In Japan, Fuse et al. compared the international reference values for Japanese and thyroid volumes of approximately 1000 schoolchildren [11]. They con- cluded that the international reference values were low compared with the data that they had obtained.

In the Fukushima Health Management Survey, an exhaustive examination revealed the reference values of thyroid volume as well as the three determinations of width, thickness, and height in Japanese children. We could update normal thyroid volume in this survey, although effects of low level radiation exposure on thyroid function including size of a thyroid gland should be carefully continuously monitored in the future [17]. In total, $71.9 \%$ of all residents aged $0-19$ years old were examined in this study, suggesting that the normal size of thyroid gland could be defined at each age or each body surface area range in Japanese children.

Ashizawa et al. demonstrated the prevalence of goiter based on the thyroid size data obtained from an epidemiological survey for investigating the prevalence of thyroid cancer conducted in Chernobyl [3]. In the study, they demonstrated the median values of thyroid volume based on approximately 110,000 children by using mechanistic equipment equipped with an ultrasonographic device. They also provided precise data of the measurement of thyroid size. In the present study, we have also demonstrated three determinations of width, thickness, and height of thyroid gland. These data may be useful for the assessment of thyroid gland volume to screen the abnormality of thyroid size, and especially for the detection of goiter in Japanese children.

The reference values in this study may be able to distinguish between normal and abnormal sized thyroid glands by using ultrasonography. However, we should note that there is no pathological evidence that thyroid glands of abnormal size detected by ultrasonography are associated with thyroid disease. There are some cases called simple goiter, which does not require treatment although the size of the thyroid gland is large. On the other hand, some goiters with enlarged thyroid can be associated with Graves' disease, autoimmune thyroiditis, or iodide-related disorders. It may be possible to clarify the frequency of the disease-associated thyroid goiter among abnormal-sized thyroid glands using the reference values obtained in this study.

The right lobe was significantly larger than the left lobe, indicating that some genetic factors may regulate thyroid volume since the prevalence of acute infectious thyroiditis due to an internal fistula extending from the piriform sinus to the thyroid as well as hemiagenesis or hypoplasia is dominant in the left lobe [18]. Another possible reason is that thyroid volume may be affected 
Table 1a The length of wide, thickness, and height of right and left thyroid lobe and the values of thyroid volume caluculated from three deteminations at the indicated age in female and male. 2.5 and 97.5 percentile were shown.

\begin{tabular}{|c|c|c|c|c|c|c|c|c|c|}
\hline \multirow{2}{*}{$\frac{\text { Female }}{\text { Age (yo) }}$} & \multicolumn{4}{|c|}{ Right lobe } & \multicolumn{4}{|c|}{ Left lobe } & \multirow{2}{*}{ Number } \\
\hline & Width (mm) & Thickness $(\mathrm{mm})$ & Height $(\mathrm{mm})$ & Volume (mL) & Width (mm) & Thickness (mm) & Height (mm) & Volume $(\mathrm{mL})$ & \\
\hline 0 & $5.4-12.0$ & $3.9-10.2$ & $10.0-26.1$ & $0.2-1.4$ & $5.7-13.1$ & $3.3-11.8$ & $9.5-25.6$ & $0.2-1.4$ & 57 \\
\hline 1 & $5.0-11.8$ & $5.1-11.3$ & $10.1-28.3$ & $0.2-1.4$ & $5.6-12.3$ & $4.6-10.7$ & $11.0-26.6$ & $0.2-1.3$ & 321 \\
\hline 2 & $5.7-12.0$ & $5.3-11.0$ & $12.4-32.1$ & $0.3-1.6$ & $5.7-12.2$ & $4.3-10.8$ & $12.9-30.5$ & $0.3-1.6$ & 488 \\
\hline 3 & $5.5-12.3$ & $5.6-11.7$ & $15.9-34.4$ & $0.4-1.8$ & $6.3-12.4$ & $4.6-10.9$ & $14.6-32.6$ & $0.4-1.6$ & 557 \\
\hline 4 & $6.4-12.8$ & $5.7-11.9$ & $17.5-36.6$ & $0.5-2.1$ & $6.7-12.9$ & $4.8-11.0$ & $17.0-33.7$ & $0.5-1.8$ & 669 \\
\hline 5 & $6.9-13.7$ & $5.8-12.0$ & $20.0-37.7$ & $0.6-2.5$ & $7.0-13.4$ & $5.0-11.1$ & $19.2-36.4$ & $0.5-2.1$ & 779 \\
\hline 6 & $7.0-14.7$ & $6.2-12.4$ & $22.0-38.6$ & $0.7-2.8$ & $7.4-14.1$ & $5.0-11.9$ & $20.5-37.9$ & $0.6-2.4$ & 772 \\
\hline 7 & $7.4-15.0$ & $6.4-12.8$ & $23.7-40.0$ & $0.9-3.1$ & $7.5-14.7$ & $5.0-11.7$ & $21.4-38.8$ & $0.6-2.7$ & 936 \\
\hline 8 & $7.7-16.3$ & $6.6-13.6$ & $25.5-40.9$ & $0.9-3.8$ & 7.9-15.4 & $5.4-12.4$ & $22.4-40.0$ & $0.7-3.0$ & 961 \\
\hline 9 & $7.9-16.5$ & $7.1-14.2$ & $26.5-42.9$ & $1.0-4.4$ & $8.0-15.5$ & $5.7-13.1$ & $23.7-42.0$ & $0.8-3.6$ & 988 \\
\hline 10 & $8.5-17.8$ & $7.4-15.0$ & $28.0-44.9$ & $1.3-4.9$ & $8.5-17.0$ & $6.2-14.0$ & $25.0-43.9$ & $1.0-4.2$ & 1075 \\
\hline 11 & 8.9-18.9 & $7.9-15.9$ & $29.5-47.0$ & $1.5-6.1$ & $9.1-18.0$ & $6.9-14.8$ & $26.5-45.6$ & $1.2-4.9$ & 1049 \\
\hline 12 & $9.7-19.8$ & $8.1-16.7$ & $29.8-47.5$ & $1.7-6.6$ & $9.6-18.4$ & $7.0-15.1$ & $26.8-46.6$ & $1.3-5.3$ & 1064 \\
\hline 13 & $9.9-19.3$ & $8.2-16.5$ & $29.2-47.7$ & $1.8-6.4$ & $9.9-18.5$ & $7.0-15.3$ & $26.9-46.8$ & $1.4-5.5$ & 1113 \\
\hline 14 & $9.9-19.9$ & $8.3-16.8$ & $30.5-47.9$ & $1.8-6.8$ & $10.3-18.8$ & $6.7-15.2$ & $27.6-46.5$ & $1.4-5.6$ & 1122 \\
\hline 15 & $10.2-20.9$ & $8.1-17.1$ & $30.2-48.2$ & $1.9-7.0$ & $10.2-19.7$ & $7.2-15.6$ & $28.9-47.3$ & $1.5-6.2$ & 1212 \\
\hline 16 & $10.3-21.3$ & $8.0-16.7$ & $31.5-48.4$ & $1.8-7.4$ & $10.3-19.4$ & $7.1-15.4$ & $28.4-47.3$ & $1.5-6.0$ & 1017 \\
\hline 17 & $10.1-20.9$ & $8.0-16.9$ & $31.8-49.1$ & $1.9-7.1$ & $10.6-19.7$ & 6.9-15.6 & $28.7-47.7$ & $1.6-6.2$ & 1170 \\
\hline 18 & $10.3-21.0$ & $8.2-16.6$ & $31.5-48.5$ & $1.8-7.0$ & $10.5-19.7$ & $7.1-15.8$ & $28.8-47.6$ & $1.7-6.0$ & 1012 \\
\hline \multirow[t]{2}{*}{19} & $10.2-22.0$ & $7.6-17.0$ & $32.1-48.2$ & $1.7-7.6$ & $10.7-20.0$ & $6.9-15.8$ & $30.2-48.0$ & $1.7-6.6$ & 632 \\
\hline & & & & & & & & Total & 16994 \\
\hline
\end{tabular}

\begin{tabular}{|c|c|c|c|c|c|c|c|c|c|}
\hline \multirow{2}{*}{$\begin{array}{l}\text { Male } \\
\text { Age (yo) }\end{array}$} & \multicolumn{4}{|c|}{ Right lobe } & \multicolumn{4}{|c|}{ Left lobe } & \multirow{2}{*}{ Number } \\
\hline & Width (mm) & Thickness $(\mathrm{mm})$ & Height $(\mathrm{mm})$ & Volume $(\mathrm{mL})$ & Width (mm) & Thickness (mm) & Height $(\mathrm{mm})$ & Volume $(\mathrm{mL})$ & \\
\hline 0 & $5.6-13.4$ & $4.9-12.4$ & $10.5-24.6$ & $0.2-1.3$ & $4.6-11.6$ & $4.0-10.9$ & $11.7-25.7$ & $0.2-1.5$ & 56 \\
\hline 1 & $5.3-12.2$ & $5.0-11.5$ & $11.1-27.7$ & $0.2-1.6$ & $5.5-12.3$ & $4.3-11.2$ & $11.1-28.2$ & $0.2-1.4$ & 368 \\
\hline 2 & $5.7-12.4$ & $5.3-11.8$ & $13.5-31.2$ & $0.3-1.6$ & $6.1-13.0$ & $4.5-11.6$ & $14.2-28.5$ & $0.4-1.5$ & 475 \\
\hline 3 & $6.1-12.2$ & $5.5-12.1$ & $15.6-33.7$ & $0.4-1.9$ & $6.7-12.7$ & $4.5-11.2$ & $15.5-31.5$ & $0.4-1.7$ & 635 \\
\hline 4 & $6.2-12.9$ & $5.4-11.8$ & $18.5-36.9$ & $0.5-2.2$ & $6.9-13.1$ & 4.6-10.9 & $17.4-34.8$ & $0.4-1.8$ & 650 \\
\hline 5 & $6.6-13.9$ & $6.1-11.7$ & $19.7-37.5$ & $0.6-2.4$ & $7.4-13.9$ & $4.8-11.0$ & $19.0-35.6$ & $0.5-2.2$ & 776 \\
\hline 6 & $7.0-14.6$ & $6.1-12.3$ & $21.1-38.0$ & $0.7-2.8$ & $7.5-14.3$ & $5.0-11.1$ & $19.3-37.0$ & $0.6-2.3$ & 822 \\
\hline 7 & $7.5-15.7$ & $6.1-12.9$ & $22.6-39.8$ & $0.8-3.2$ & $7.9-15.1$ & $5.3-11.4$ & $21.6-38.3$ & $0.7-2.5$ & 985 \\
\hline 8 & $7.8-16.4$ & $6.7-13.5$ & $24.5-40.8$ & $0.9-3.6$ & $8.2-15.6$ & $5.5-12.4$ & 21.7-39.8 & $0.8-3.0$ & 1046 \\
\hline 9 & $8.4-17.2$ & $6.6-13.6$ & $25.8-42.2$ & $1.1-4.0$ & $8.7-16.2$ & $5.5-12.4$ & $22.9-40.7$ & $0.9-3.2$ & 1008 \\
\hline 10 & $8.4-17.9$ & $6.9-14.6$ & $27.2-44.3$ & $1.1-4.8$ & $8.5-16.8$ & $5.9-12.8$ & $23.9-42.1$ & $0.9-3.8$ & 1080 \\
\hline 11 & $9.0-18.9$ & $7.1-15.3$ & $27.3-45.7$ & $1.3-5.4$ & $9.0-17.6$ & $5.9-14.3$ & $24.7-44.3$ & $1.0-4.7$ & 1134 \\
\hline 12 & $9.3-19.1$ & $7.6-16.5$ & $27.9-46.7$ & $1.5-6.1$ & $9.8-18$ & $6.7-14.6$ & $26.1-46.0$ & $1.2-5.0$ & 1115 \\
\hline 13 & $9.8-19.8$ & 8.4-16.9 & $29.6-47.4$ & $1.8-6.3$ & $9.9-18.0$ & $7.2-15.3$ & $26.6-46.6$ & $1.4-5.4$ & 1040 \\
\hline 14 & $10.5-20.3$ & $8.7-17.5$ & $31.6-48.2$ & $2.1-7.3$ & $10.2-19.3$ & $7.4-16.0$ & 27.7-47.2 & $1.5-6.0$ & 1116 \\
\hline 15 & $10.6-21.5$ & $9.0-17.6$ & $31.6-49.6$ & $2.2-7.7$ & $10.8-19.6$ & $7.6-16.0$ & $29.4-48.1$ & $1.7-6.2$ & 1164 \\
\hline 16 & $10.4-21.9$ & 8.9-18.6 & $32.5-50.6$ & $2.2-8.1$ & $10.6-20.0$ & $7.5-16.4$ & 29.1-48.6 & $1.7-6.9$ & 1089 \\
\hline 17 & $10.9-22.9$ & $9.0-18.6$ & $32.0-49.6$ & $2.2-8.4$ & $11.0-20.2$ & $8.0-16.5$ & $29.0-48.3$ & $1.9-6.8$ & 1140 \\
\hline 18 & $11.4-22.5$ & $8.9-18.8$ & $34.1-51.6$ & $2.5-8.5$ & $11.8-20.1$ & $8.1-16.8$ & $30.7-50.2$ & $2.0-7.1$ & 980 \\
\hline \multirow[t]{2}{*}{19} & $10.9-22.4$ & $9.2-18.5$ & 33.3-51.1 & $2.4-8.5$ & $11.4-20.2$ & $8.2-16.7$ & $30.2-49.0$ & $2.0-7.1$ & 554 \\
\hline & & & & & & & & Total & 17233 \\
\hline
\end{tabular}


Table 1b The length of wide, thickness, and height of right and left thyroid lobe and the values of thyroid volume caluculated from three deteminations at the indicated range of body surface area in female and male. 2.5 and 97.5 percentile were shown.

\begin{tabular}{|c|c|c|c|c|c|c|c|c|c|}
\hline \multirow{2}{*}{$\begin{array}{l}\text { Female } \\
\text { Body surface } \\
\text { area }\left(\mathrm{m}^{2}\right)\end{array}$} & \multicolumn{4}{|c|}{ Right lobe } & \multicolumn{4}{|c|}{ Left lobe } & \multirow[b]{2}{*}{ Number } \\
\hline & \multicolumn{4}{|c|}{ Width $(\mathrm{mm})$ Thickness $(\mathrm{mm})$ Height $(\mathrm{mm})$ Volume $(\mathrm{mL})$} & \multicolumn{4}{|c|}{$\overline{\text { Width }(\mathrm{mm}) \text { Thickness }(\mathrm{mm}) \text { Height }(\mathrm{mm}) \text { Volume }(\mathrm{mL})}$} & \\
\hline 0.3 & $5.4-$ & $4.9-$ & $12.7-$ & $0.3-$ & $5.5-$ & $7.1-$ & $12.8-$ & $0.4-$ & 3 \\
\hline 0.4 & $5.0-11.7$ & $4.9-11.1$ & $10.2-30.3$ & $0.2-1.5$ & $5.3-12.3$ & 4.1-10.9 & $10.2-27.0$ & $0.2-1.2$ & 233 \\
\hline 0.5 & $5.6-11.9$ & $5.3-11.4$ & $11.6-30.8$ & $0.3-1.6$ & $5.9-12.2$ & 4.3-10.6 & $12.7-29.4$ & $0.3-1.4$ & 554 \\
\hline 0.6 & $5.8-12.3$ & $5.6-11.6$ & $15.3-34.9$ & $0.4-2.0$ & $6.4-12.7$ & $4.6-10.9$ & $14.6-33.4$ & $0.4-1.7$ & 866 \\
\hline 0.7 & $6.7-13.4$ & $6.0-11.9$ & $18.5-37.0$ & $0.6-2.3$ & 6.9-13.0 & $5.0-11.0$ & $18.2-35.9$ & $0.5-2.0$ & 1059 \\
\hline 0.8 & $7.0-14.4$ & $6.2-12.4$ & $21.9-38.8$ & $0.7-2.8$ & 7.4-13.9 & $5.0-11.5$ & $21.2-37.4$ & $0.6-2.3$ & 1185 \\
\hline 0.9 & $7.6-15.4$ & $6.4-12.9$ & $24.8-40.0$ & $0.9-3.2$ & $7.7-14.8$ & $5.1-12.0$ & $22.2-39.4$ & $0.7-2.8$ & 1193 \\
\hline 1.0 & $8.1-16.2$ & 7.1-13.9 & $25.8-41.7$ & $1.1-4.0$ & $8.2-15.4$ & $6.0-12.7$ & $22.4-40.4$ & $0.8-3.2$ & 1142 \\
\hline 1.1 & $8.4-17.2$ & $7.5-14.7$ & $27.8-44.2$ & $1.2-4.5$ & $8.5-16.5$ & $6.0-13.9$ & 24.4-43.2 & $1.0-3.9$ & 959 \\
\hline 1.2 & $9.1-17.8$ & $7.7-15.6$ & $28.1-45.3$ & $1.4-5.0$ & $9.0-17.0$ & $6.7-14.5$ & $25.2-44.2$ & $1.1-4.4$ & 1025 \\
\hline 1.3 & $9.5-19.2$ & $8.0-16.1$ & $29.2-46.9$ & $1.6-5.9$ & $9.6-18.3$ & $6.9-15.0$ & $26.7-45.7$ & $1.3-5.1$ & 1586 \\
\hline 1.4 & $9.7-19.9$ & $8.0-16.3$ & $30.6-47.5$ & $1.8-6.4$ & $10.0-18.6$ & $7.0-15.0$ & $27.9-46.6$ & $1.5-5.3$ & 2635 \\
\hline 1.5 & $10.4-20.6$ & $8.2-16.9$ & $31.5-48.2$ & $1.9-6.9$ & $10.6-19.5$ & $7.0-15.3$ & $28.7-47.5$ & $1.6-5.9$ & 2561 \\
\hline 1.6 & $10.6-21.3$ & 8.3-17.0 & $32.3-49.6$ & $2.0-7.5$ & $10.8-20.0$ & $7.3-15.8$ & $29.2-47.9$ & $1.7-6.3$ & 1346 \\
\hline 1.7 & $11.0-21.5$ & $8.6-17.6$ & $31.7-48.8$ & 2.3-7.7 & $11.0-20.9$ & $7.5-16.5$ & $30.0-47.8$ & $1.9-6.8$ & 424 \\
\hline 1.8 & $10.7-22.7$ & 7.9-18.8 & $29.2-48.5$ & $1.7-9.6$ & $10.7-20.9$ & $7.7-18.8$ & $25.9-48.2$ & $1.4-8.9$ & 166 \\
\hline 1.9 & 7.6- & 8.7- & $4.7-$ & $0.7-$ & 8.9- & 5.3- & 19.4- & $0.7-$ & 39 \\
\hline 2.0 & 11.8- & 8.8- & 31.5- & $1.7-$ & 9.6- & 7.7- & $28.1-$ & $1.1-$ & 14 \\
\hline 2.1 & 18.0- & 12.8- & 40.3- & $5.0-$ & $16.2-$ & $14.2-$ & 36.6- & 4.4- & 3 \\
\hline 2.2 & NA & NA & NA & NA & NA & NA & NA & NA & 0 \\
\hline \multirow[t]{2}{*}{2.3} & NA & NA & NA & NA & NA & NA & NA & NA & 1 \\
\hline & & & & & & & & Total & 16994 \\
\hline
\end{tabular}

\begin{tabular}{|c|c|c|c|c|c|c|c|c|c|}
\hline \multirow{2}{*}{$\begin{array}{l}\text { Male } \\
\text { Body surface } \\
\text { area }\left(\mathrm{m}^{2}\right)\end{array}$} & \multicolumn{4}{|c|}{ Right lobe } & \multicolumn{4}{|c|}{ Left lobe } & \multirow[b]{2}{*}{ Number } \\
\hline & \multicolumn{4}{|c|}{ Width (mm) Thickness (mm) Height (mm) Volume $(\mathrm{mL})$} & \multicolumn{4}{|c|}{ Width $(\mathrm{mm})$ Thickness $(\mathrm{mm})$ Height $(\mathrm{mm})$ Volume $(\mathrm{mL})$} & \\
\hline 0.2 & 7.9 & 7.4- & 21.5 & $0.7-$ & $9.2-$ & $5.6-$ & $22.7-$ & $0.6-$ & 2 \\
\hline 0.3 & $5.7-$ & $5.7-$ & $12.2-$ & $0.2-$ & $5.7-$ & $6-$ & $13.8-$ & $0.3-$ & 4 \\
\hline 0.4 & $5.3-12.7$ & $4.8-11.2$ & $11.1-27.8$ & $0.2-1.5$ & $5.0-12.1$ & $4.1-10.9$ & $11.3-26.4$ & $0.2-1.4$ & 179 \\
\hline 0.5 & $5.6-12.5$ & $5.1-11.5$ & $12.3-29.3$ & $0.3-1.6$ & $5.8-12.8$ & $4.5-11.3$ & $12.9-28.1$ & 0.3-1.5 & 538 \\
\hline 0.6 & $6.1-12.4$ & $5.3-12.0$ & $14.7-34.1$ & $0.4-1.9$ & $6.7-12.7$ & $4.4-11.2$ & $15.4-31.0$ & $0.4-1.6$ & 891 \\
\hline 0.7 & $6.4-13.1$ & $5.7-11.8$ & $18.5-36.9$ & $0.5-2.2$ & $7.1-13.2$ & $4.7-11.0$ & $17.8-35.0$ & $0.5-1.9$ & 1058 \\
\hline 0.8 & $6.9-14.7$ & 6.1-12.0 & $21.1-38.8$ & $0.7-2.7$ & $7.5-14.3$ & $5.0-11.0$ & $20.0-37.2$ & $0.6-2.3$ & 1208 \\
\hline 0.9 & $7.6-15.6$ & $6.2-13.2$ & $23.0-39.7$ & $0.8-3.3$ & $8.1-15.1$ & $5.3-11.6$ & $21.3-38.4$ & $0.7-2.6$ & 1208 \\
\hline 1.0 & $8.1-16.5$ & $6.7-13.0$ & $24.9-41.3$ & $1.0-3.5$ & $8.5-15.9$ & $5.6-12.4$ & $22.4-40.0$ & $0.8-3.0$ & 1269 \\
\hline 1.1 & $8.3-17.1$ & $7.0-14.1$ & $26.3-42.5$ & $1.1-4.1$ & $8.7-16.2$ & $5.7-13.0$ & $23.4-41.0$ & $0.9-3.5$ & 1156 \\
\hline 1.2 & 8.9-18.8 & $7.2-15.0$ & $28.4-44.5$ & $1.3-4.9$ & $8.5-16.6$ & $6.1-13.4$ & $24.7-42.5$ & $1.0-3.9$ & 1030 \\
\hline 1.3 & $9.2-18.6$ & $7.6-15.4$ & $28.9-45.6$ & $1.4-5.3$ & $9.3-17.5$ & $6.5-14.3$ & $26.1-44.4$ & $1.1-4.6$ & 964 \\
\hline 1.4 & $9.5-19.2$ & $7.6-16.7$ & $27.8-46.8$ & $1.6-6.0$ & $9.7-18.0$ & $6.6-15.0$ & $26.4-45.6$ & $1.2-5.2$ & 975 \\
\hline 1.5 & $10.0-19.8$ & 8.6-16.7 & $30.0-47.5$ & $1.9-6.7$ & $9.9-18.6$ & $7.4-15.2$ & $27.4-46.8$ & $1.5-5.7$ & 1315 \\
\hline 1.6 & $10.3-20.6$ & $8.6-17.7$ & $32.2-49.0$ & $2.1-7.3$ & $10.6-19.1$ & $7.6-15.8$ & $29.0-47.7$ & $1.7-6.1$ & 1787 \\
\hline 1.7 & $11.0-20.9$ & $9.1-17.8$ & $31.0-49.6$ & $2.4-7.5$ & $11.0-19.3$ & $7.9-16.1$ & $29.4-48.4$ & $1.8-6.2$ & 1628 \\
\hline 1.8 & $11.3-22.4$ & $9.2-18.3$ & $33.6-51.5$ & $2.5-8.5$ & $11.6-20.2$ & $7.9-16.6$ & $29.6-49.4$ & $2.0-7.0$ & 1124 \\
\hline 1.9 & $11.7-24.3$ & $9.2-18.7$ & $33.4-51.6$ & $2.6-8.9$ & $12.0-21.0$ & $8.4-17.0$ & $29.5-49.2$ & $2.0-7.4$ & 503 \\
\hline 2.0 & $12.3-24.4$ & $10.3-20.1$ & $32.5-54.6$ & $2.9-10.9$ & $11.4-21.4$ & $8.0-18.0$ & $30.6-51.4$ & $2.2-8.2$ & 245 \\
\hline 2.1 & $13.5-25.9$ & $10.1-20.1$ & $33.5-53.2$ & $2.8-10.0$ & $11.3-23.4$ & $7.9-18.5$ & $26.7-51.4$ & $1.8-7.9$ & 85 \\
\hline 2.2 & $7.4-27.8$ & 7.9-21.3 & $26.1-51.0$ & $1.0-14.6$ & $10.6-21.8$ & $8.2-19.2$ & $27.1-51.3$ & $1.3-10.4$ & 42 \\
\hline 2.3 & 8.3- & 11.5- & 33.3- & $1.9-$ & 7.9- & 8.9- & 29.9- & $1.7-$ & 16 \\
\hline 2.4 & $15.7-$ & $14.8-$ & $33.2-$ & $4.2-$ & $16.9-$ & 9.9- & 39.3- & $3.4-$ & 3 \\
\hline 2.5 & $16.2-$ & 10.3- & 36.6- & $3.2-$ & 16.0- & 11.8- & $34.5-$ & $3.7-$ & 2 \\
\hline 2.6 & NA & NA & NA & NA & NA & NA & NA & NA & 1 \\
\hline & & & & & & & & Total & 17233 \\
\hline
\end{tabular}



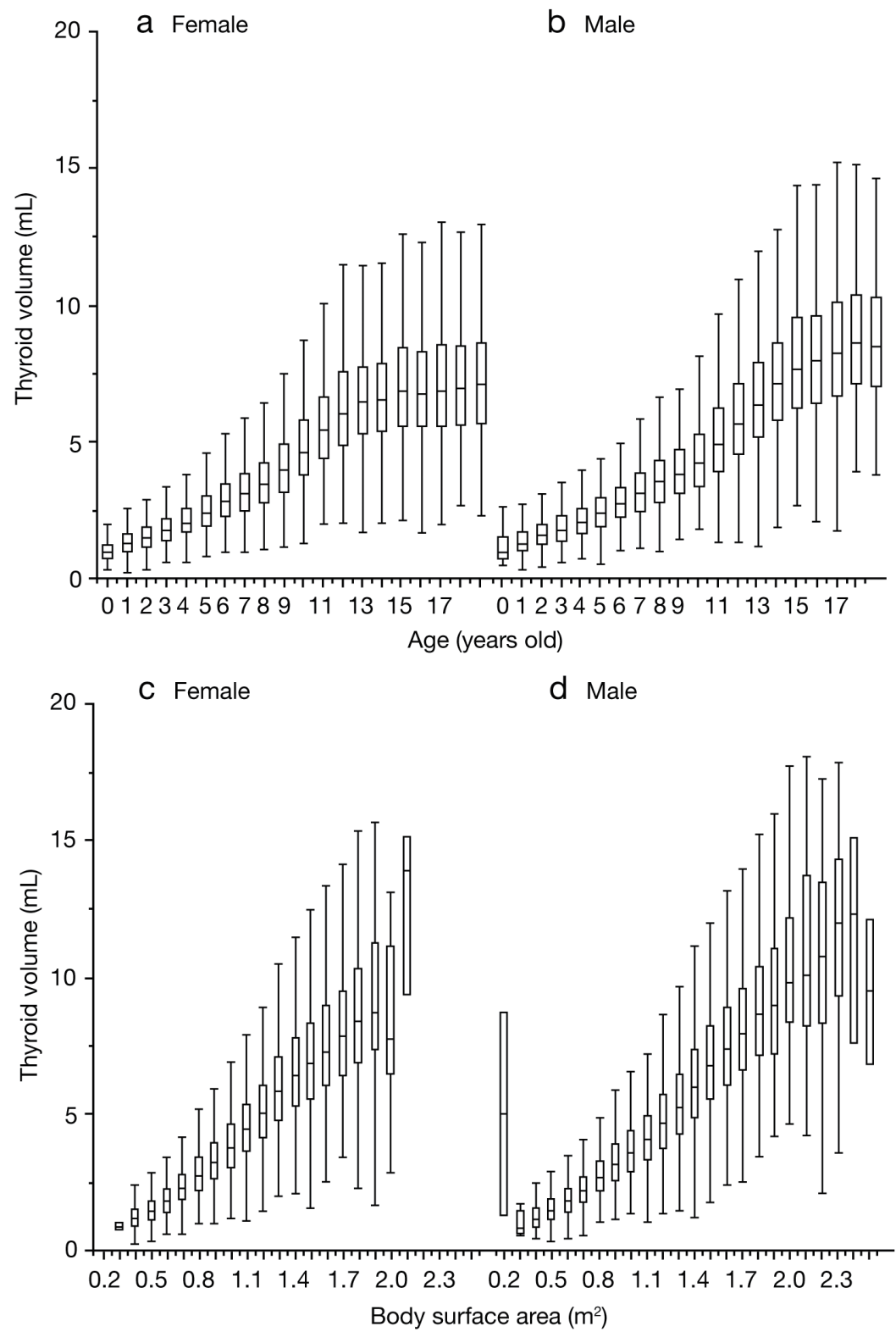

Fig. 1 Box-and-whisker plots showing distributions of thyroid volume in each age, gender, and range of body surface area.

Width, thickness and height of each lobe were measured and the volume of each lobe was calculated by the mean of the elliptical shape volume formula $(\pi / 6 \times$ width $\times$ thickness $\times$ height $)$. The sum volume of both lobes represents the total thyroid volume. The boxes represent the $25^{\text {th }}$ and $75^{\text {th }}$ percentiles, and the horizontal lines within the box represent median values. The whiskers represent the $10^{\text {th }}$ and $90^{\text {th }}$ percentiles, respectively. Each value was plotted by age in female (a) and in male (b), as well as by body surface area in female (c) and in male (d).

Table 2 Compairson of thyroid size between right and left lobe (n; 34227)

\begin{tabular}{lccc}
\hline & Right & Left & $p$ value \\
\hline Width $(\mathrm{mm})$ & $12.8 \pm 3.3$ & $12.7 \pm 2.9$ & $<0.001$ \\
Thickness $(\mathrm{mm})$ & $11.0 \pm 2.7$ & $9.7 \pm 2.5$ & $<0.001$ \\
Height $(\mathrm{mm})$ & $35.9 \pm 7.3$ & $34.2 \pm 7.3$ & $<0.001$ \\
Volume $(\mathrm{mL})$ & $2.9 \pm 1.7$ & $2.4 \pm 1.4$ & $<0.001$ \\
\hline
\end{tabular}

Table 3 The difference of thyroid size in female and male

\begin{tabular}{lccccc}
\hline & Number & $\begin{array}{c}\text { Age (years) } \\
\text { mean } \pm \text { SD }\end{array}$ & $\begin{array}{c}\mathrm{BSA}^{\mathrm{a}}\left(\mathrm{m}^{2}\right) \\
\text { mean } \pm \mathrm{SD}\end{array}$ & $\begin{array}{c}\text { Thyroid } \\
\text { volume }(\mathrm{mL}) \\
\text { mean } \pm \mathrm{SD}\end{array}$ & $\begin{array}{c}\mathrm{ANCOVA}^{\mathrm{b}} \\
\text { mean }(\mathrm{mL}) 95 \% \\
\text { confidence interval }\end{array}$ \\
\hline Female & 16994 & $11.0 \pm 4.9$ & $1.17 \pm 0.35$ & $5.2 \pm 2.9$ & $5.4(5.396-5.453)$ \\
Male & 17233 & $10.9 \pm 4.9$ & $1.25 \pm 0.42$ & $5.4 \pm 3.1$ & $5.2(5.208-5.265)$ \\
$p$-value & 0.646 & $<0.001$ & $<0.001$ & $<0.001^{\mathrm{c}}$ \\
\hline
\end{tabular}

${ }^{\mathrm{a}}$, BSA, body surface area; ${ }^{\mathrm{b}}$, ANCOVA, analysis of covariance; ${ }^{\mathrm{c}}$, Value- $p$ refers to ANCOVA, adjusted for BSA 
by surrounding organs such as the esophagus, which is almost always present on the left side.

Thyroid volume in female is preferentially increased after correction of body surface area compared with that in male, suggesting that gender-related factors including sex hormones may be related to the development and growth of the thyroid gland.

The accuracy of the measurement itself may have three limitations. Firstly, an accurate thyroid volume may not be determined by the three dimensions of width, thickness, and height, as a thyroid lobe does not have a perfect elliptical shape. The volume is calculated using the standard formula for ovoids such as $\pi / 6 \times$ width $\times$ thickness $\times$ height as described in some articles including ours. Brunn et al., however, applied 0.479 as an optimized correction factor instead of $\pi / 6$ [19]. Secondly, isthmus volume was not included in "thyroid volume" in this study. Thirdly, the measurement of thyroid size, especially the height of thyroid lobe, may not have been accurate when the length of the height was too long to be measured in one scan of B-mode. On the contrary, the infant's neck was too short to attach the probe completely. In addition, ultrasound examination itself may have caused a sensation of fear in babies, indicating that it may be difficult for small children to be examined precisely in a brief ultrasound screening.

In conclusion, the present study reported updated references of thyroid volume using ultrasonography through the Fukushima Health Management Survey. The right lobe was statistically larger than the left, and thyroid volume was dominant in female. These reference values may contribute to the ultrasonographic diagnosis of thyroid goiter and hypoplasia, although further study is required.

\section{Acknowledgements}

We express our gratitude to all members participating in the Fukushima Health Management Survey. We also thank to Ms. Tazuko Kawasaki and Ms. Takako Takahashi for their excellent secretarial assistance.

The findings and conclusions of this article are solely the responsibility of the authors and do not represent the official views of the Fukushima Prefectural Government.

\section{Appendix}

Other Participating Expert Committee Members, Advisors and Staffin TheFukushimaHealth Management Survey: Kenji Kamiya, Seiji Yasumura, Kenneth E. Nollet, Kumiko Tsuboi, Shiro Matsui, Masaharu Maeda, Shigeatsu Hashimoto, Keiya Fujimori, Suguru Ishida, Hideto Takahashi, Tetsuo Ishikawa, Akira Sakai, Yuko Hino, Hiroshi Mizunuma, Keiichi Nakano, Hirokazu Okayama, Chiyo Ohkouchi, Tomomi Hakoiwa, Chisato Takahashi, Yukari Sato, Ayako Sato, Nobuko Sakuma, Toshie Sakagami, Manabu Ohishi, Norikazu Abe, Masao Kuribara, Masahiko Henmi, Takao Yamahata, Mizuki Sekino, and Yuko Sato.

\section{Grants}

This survey was conducted as part of Fukushima Prefecture's post-disaster recovery plans and was supported by the national "Health Fund for Children and Adults Affected by the Nuclear Incident".

\section{Disclosure Statement}

The authors have nothing to disclose. There is no conflict of interest in this study.

\section{References}

1. Kharchenko VP, Kotlyarov PM, Mogutov MS, Alexandrov YK, Sencha AN, et al. (2010) Ultrasound examination of the thyroid gland in children. In: Kharchenko VP, Kotlyarov PM, Mogutov MS, Alexandrov YK, Sencha AN, Patrunov YN, Belyaev DV. Ultrasound Diagnosis of Thyroid Diseases. Springer-Verlag, Berlin, Heidelberg: 35-45.

2. Parshin VS, Yamashita S, Tsyb AF (2013) Ultrasound criteria of assessment of normal thyroid gland. In: Parshin VS, Yamashita S, Tsyb AF, Ultrasound
Diagnosis of Thyroid Diseases in Russia; Saenko V, Suzuki S (eds), Obninsk, Nagasaki: 12-22.

3. Ashizawa K, Shibata Y, Yamashita S, Namba H, Hoshi M, et al. (1997) Prevalence of goiter and urinary iodine excretion levels in children around Chernobyl. J Clin Endocrinol Metab 82: 3430-3433.

4. Skyabin AM, Drozdovitch V, Belsky Y, Leshcheva SV, Mirkhaidarov AK, et al. (2010) Thyroid mass in children and adolescents living in the most exposed areas to Chernobyl fallout in Belarus. Radiat Prot Dosimetry 
142: 292-299.

5. Ueda D (1990) Normal volume of the thyroid gland in children. J Clin Ultrasound 18: 455-462.

6. Yasumura S, Hosoya M, Yamashita S, Kamiya K, Abe M, et al. (2012) Study protocol for the Fukushima Health Management Survey. J Epidemiol 22: 375-383.

7. Chanoine JP, Toppet V, Lagasse R, Spehl M, Delange F (1991) Determination of thyroid volume by ultrasound from the neonatal period to late adolescence. Eur J Pediatr 150: 395-399.

8. [No authors listed] (1997) Recommended normative values for thyroid volume in children aged 6-15 years. World Health Organization \& International Council for Control of Iodine Deficiency Disorders. Bull World Health Organ 75: 95-97.

9. Delange F, Benker G, Caron P, Eber O, Ott W, et al. (1997) Thyroid volume and urinary iodine in European schoolchildren: standardization of values for assessment of iodine deficiency. Eur J Endocrinol 136: 180187.

10. Zimmermann MB, Hess SY, Molinari L, De Benoist B, Delange F, et al. (2004) New reference values for thyroid volume by ultrasound in iodine-sufficient schoolchildren: a World Health Organization/ Nutrition for Health and Development Iodine Deficiency Study Group Report. Am J Clin Nutr 79: 231-237.

11. Fuse Y, Saito N, Tsuchiya T, Shishiba Y, Irie M (2007) Smaller thyroid gland volume with high urinary iodine excretion in Japanese schoolchildren: normative reference values in an iodine-sufficient area and comparison with the WHO/ICCIDD reference. Thyroid 17: 145-
155.

12. Liu Y, Huang H, Zeng J, Sun C (2013) Thyroid volume, goiter prevalence, and selenium levels in an iodine-sufficient area: a cross-sectional study. BMC Public Health 13: 1153.

13. Kim BK, Choi YS, Oak CH, Park YH, Kim JH, et al. (2012) Determination of thyroid volume by ultrasonography among schoolchildren in Philippines. Int $J$ Endocrinol 2012: 387971.

14. Zou Y, Ding G, Lou X, Zhu W, Mao G, et al. (2013) Factors influencing thyroid volume in Chinese children. Eur J Clin Nutr 67: 1138-1141.

15. Langer P, Tajtakova M, Kocan A, Drobna B, Kostalova L, et al. (2012) Thyroid volume, iodine intake, autoimmune thyroid disorders, inborn factors, and endocrine disruptors: twenty-year studies of multiple effects puzzle in Slovakia. Endocr Regul 46: 191-203.

16. Abd El Naser Yamamah G, Kamel AF, Abd-El Dayem S, Hussein AS, Salama H (2013) Thyroid volumes and iodine status in Egyptian South Sinai schoolchildren. Arch Med Sci 9: 548-554.

17. Yamashita S, Suzuki S (2013) Risk of thyroid cancer after the Fukushima nuclear power plant accident. Respir Investig 51: 128-133.

18. Miyauchi A, Matsuzuka F, Takai S, Kuma K, Kosaki G (1981) Piriform sinus fistula. A route of infection in acute suppurative thyroiditis. Arch Surg 116: 66-69.

19. Brunn J, Block U, Ruf G, Bos I, Kunze WP, et al. (1981) Volumetric analysis of thyroid lobes by real-time ultrasound (author's transl). Dtsch Med Wochenschr 106: 1338-1340 (In German). 